\title{
The Functional Value of the Participle in a Patent Text
}

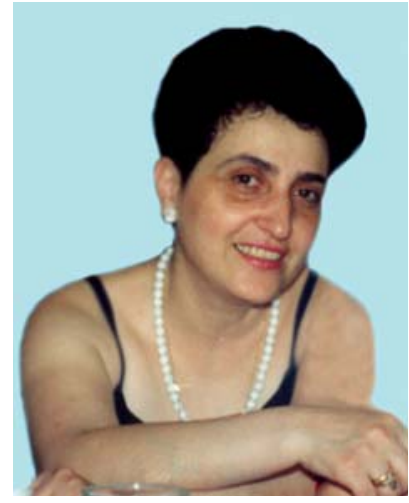

Naira Gasparyan

$7 \Gamma$ he present research deals with the participle in the American patent text identified as Patent Specification. The official character of the detailed description of the invention is provided by different linguostylistic means - phenomena which are not usually available in official documents in the strictest sense of the word. The present study of patent specifications has revealed a completely new and unique field of linguistic research that explores the complex correlation and intertwining of the stylistic elements, functionally characteristic of scientific discourse and official documents.

The legally established structural features of a patent determine the stylistic value of language units in Patent Specification. The use of the participle emphasises the logical coherence of the text under discussion. A more profound examination of the participle makes the motivation for our choice of subject matter absolutely clear. As has already been established, the participle is, according to frequency of use, one of the most prominent language units in a patent text and plays an important part in text organization.

The task of the present report is to show that the participle in a patent text is very often a stylistically coloured element. It should be noted that the whole layout of a patent text is represented in several conventionally agreed chapters. The way a Patent Specification is compiled and the way a patent text is organized generally can already be viewed as a linguostylistic peculiarity.

The Aristotelian maxim that "every whole is a unity of a beginning, a main part and an end" proves to the point when applied to the field of text. The process of perceiving a text as a whole can be successfully realized if it is viewed as a unity of all its parts the title, the beginning, the main body, and the end. They focus the reader's attention on the most characteristic features of the object or phenomenon described.

The first page of a patent, arranged in a universally accepted way, contains information about the country, the sphere of the invention, the application date, the field of research, the references cited, other publications, information about the experts who checked the existence of novelty, the registration number of the patent, the abstract (which is written according to the usual rules for writing a good scientific abstract), etc.2

Every Patent Specification consists of certain parts with subheadings dealing with the technical field, the background knowledge of the field, and the description of the 
invention. As a rule, the closing passage of the final part contains claims and is saturated with information, since it is the concluding part of a patent that reveals the author's actual desire to be granted the monopoly of the invention.

The patent heading is the shortest and most informative part of the text. For this sole reason, it is common for several noun attributes to be piled up before the noun they qualify. In the heading of a patent, the participle emphasises certain essential qualities of the object invented.

1. Sensitized Epitaxial Infrared Detector 4, 447, 446;

2. Graded Bandgap Multilayer Avalanche Photodetector with Energy Step Backs 4, 476, 477;

3. Avalanche Photodetector Including Means for Separating Electrons and Holes 4, 486, 765.

In the first two examples, the forms of the past participle sensitized and graded stress the qualities that have been improved.

In the third example the present participle including does not emphasise a quality that the proposed avalanche photodetector possesses but, instead, it prepares the reader to concentrate on the idea that follows it, that is, means for separating electrons and holes.

The study of the above-mentioned examples shows that the use of the participle in a patent heading is connected with the disclosure of the gist of the invention.

As is seen from the examples above, very often the participle appears to complete the information conveyed by the key noun of the utterance. The requirement that the heading should be made specific is justified because special catalogues are compiled from patent headings, enabling specialists to carry out thematic searches in patent libraries.

Participles appearing in the chapter 'Technical Field' complete the description of the object invented or of the field to which the invention pertains.

If the text of a patent begins with an introductory chapter, 'Cross Reference to Related Application', the participle related forms a patent term together with the noun application and carries significant information. Its presence in the text suggests that the previously existing patent dealing with the same invention has lost its power. The sentence following the subheading states this fact. Additionally, the participle abandoned beside the patent number in this introductory passage indicates this.

A thorough observation of all chapters of a patent text proves that the use of the participle is either connected with the discussion of the existing properties of the matter under observation, or with the new and advantageous characteristics of the invention.

1. The present invention relates generally to semiconductor devices and more particularly to photodetectors intended for use in the infrared region...

2. ... a number of different types of devices for use have been proposed for different applications requiring different combinations of size, image, quality... 
3. Yet another object is to provide an $X-Y$ addressed array of infrared detector devices requiring only one gate electrode per line of such devices.

Some authors, in order to stress this or that particular feature or aspect of their invention, make use of inversion. This stylistic-syntactic device has a primary purpose to indicate the part of the object in which the advantageous changes (fundamental or partial) have taken place. This, on the other hand, gives the author a chance to establish a contact with his expected reader, draw the attention of the latter to the novelty of his invention and convince him in the privilege of the object he offers to him.

a) In a semiconductor avalanche photodetector...

b) In accordance with the principles of the present invention...

c) In a particular embodiment of the invention...

d) In an alternative possible mode of operation...

The above-mentioned examples of inversion contain no participle, since the latter generally does not appear when particular details about the object under consideration need to be mentioned.

Participle I often found in participial clauses, mainly attributive, serves as a linking element. This phenomenon is known as definitization and characterizes scientific and technical writing. The frequent appearance of participial clauses in this type of texts can be explained in the following way. Linking elements like 'that' and 'which' do not carry any scientific or technical information. Moreover, they appear to separate the participial clause from the main clause. 'That' and 'which' subordinate clauses drive the rheme of the sentence far from the theme and, hence, distract the reader's attention from the core issue, while the participial clause introduces the rheme without delay.

... It (the gate electrode) forms a fringing field extending into the detection area which further facilitates the conduction of the excited electrons in the detection areas into the gate region...

Separating the given passage into minimal perspective units, we shall obtain the following:

It (the gate electrode) forms a fringing field extending into the detection area which further facilitates the conduction of the excited electrons in the detection area into the gate region
Theme

Rheme1

Rheme2

Rheme3 
As can be observed in the example above, the two parts of the same idea (Rheme1; Rheme 2) follow each other directly. The participle extending introduces the whole idea at once, without delay; the noun field is not separated from the idea extending into the detection area by unnecessary wh- connectives.

Attributive clauses with wh- connectives help the reader to keep his attention on the issue longer, thus giving him a little more time for further consideration. The 'which' clause (Rheme 3) in the above-mentioned sentence is no less important from the point of view of bearing scientific information, and actually it is the logical continuation of the participial clause. The observation shows that the specifically important information is delivered through a wh- clause whose connection with the main clause is looser, while the connection of the participial clause with the main clause is tighter.

In a patent text the participle is also found in a compositional pattern of syntactical arrangement known as parallel construction in which the repetition of a certain language unit, the participle in this case, backs up the parallel construction. The use of the participle in factological writings in general, and in a patent text in particular, carries the idea of semantic equality of the parts of the construction. In the text of a patent some parts of successive sentences or clauses are repeated in a parallel arrangement, as in the following articles of the Patent Formula (patent 4.486,765) (from 11 to 17):

11. A device as recited in claim 10 in which...

13. A device as recited in claim 12 comprising...

14. A device as recited in claim 12 or $13 . .$.

15. A device as recited in claim 14 wherein...

16. A device as recited in claim $15 . .$.

17. A device as recited in claim 16...

This kind of syntactic (compositional) pattern appears after the expressions that introduce the claims (Claims; I claim; We claim; What we claim is; What is claimed is). These phrases, always followed by a colon, introduce the novelty of the invention and have the core information at the top of the pattern, whose language cannot be imagined without participles. The whole pattern makes one grammatically independent system and should be viewed as one syntactic whole (a supra-phrasal unit).

The participle in a syntactic parallel construction backs up perfection of expression in a Patent Formula, while participial clauses in the part of the text appearing before the claims are intended for a more economical organization of the information.

Syntactic parallelism is traditionally used to enumerate this or that group of facts or arguments. Its use in a Patent Formula (the closing section of a patent text $\mathrm{t}^{1}$ ) is a norm which aims at attracting and focusing the reader's attention on the most important part of the text - the novelty of the invention. The legal significance of the document is provided by the syntactic and semantic layout of the Formula. ${ }^{2}$ The syntactic-stylistic analysis of a Patent Formula suggests that in this part, 
particularly, we deal with another style of Intellective Prose - the style of business documents.

The research shows that the participle can be found 1) in expressions established as patent terms, such as 'related application, 'those skilled in the art'; 2) in those parts of a patent text which deal either with the detailed description of the object invented, or with the advantages of the new invention discussed in contrast to the main disadvantage of the previously existing object; 3) in participial attributive clauses, when the flux of information needs to be directed to the reader without delay; 4) in the concluding part of the text (Patent Formula), which is compiled so that Participle I is the cornerstone of primary articles and the Participle II is that of supplementary articles ${ }^{3}$.

The linguistic structures in which the participle is used makes one aware of language as a sequentially organized communication system in which emphasising may be important for the proper understanding of the message and its implications.

Our investigation has shown that in the case of the American patent text, participial clauses appear even more frequently as the patent law of the United States requires the full and precise discussion of each topic, which, in turn, presupposes the use of longer sentences (complex, compound and complex-compound).

In the text under study, attributive clauses are introduced by the participle since connecting words, not informative by nature, weaken the bond between the main clause and the subordinate clause. The use of the participle makes the linkage smooth and the information direct and more vivid.

The structure of the patent presents a logical sequence of sentences combined into paragraphs adhering in the most accurate way, so that the layout of the facts in the text leaves no place for ambiguity. The research has shown that the participle is abundantly used in patent specifications and although there are many other structural elements, the participle (I and II) is the one that can never be substituted by other structural means.

However, our thorough observations have proved that each particular case of the participle has some additional stylistic and pragmatic value motivated by the logical and legal purpose of a patent text.

\section{References and Notes:}

1. Many authors have touched on the importance of the concluding part of a literary work, but the concluding part of a patent text has not been previously mentioned as a separate type. Our investigation has revealed that there is no discussion in the literature on the stylistic-semantic value of a Patent Formula, or of its pragmatic and stylistic functions. The establishment of its pragmatic and stylistic functions will help to clarify and understand the whole text as one inseparable unit. The logical arrangement of a Patent Formula reminds the reader of a summary, but its stylistic-semantic expression is unique. It seems to resemble a mathematicallogical sequence of thoughts compiled to meet the strict requirements of the patent law of the United States. 
2. On the structure of American Patent Specifications see: Ø åï åëåâ È.Ï . Структура патентных описаний/Труды ЦНИИПИ, М., 1967.

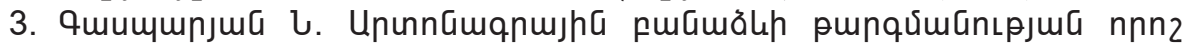
unuGăGuhmunlnspjnıGGitn, Uuunnhl, 1998:

\section{Participle-h qnnounulyuG undtpp ununnGuqph untpuunnıu}

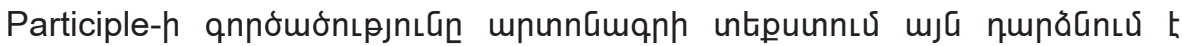

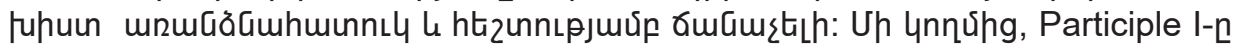

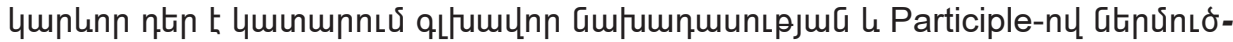

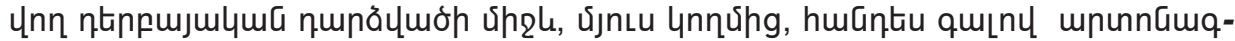

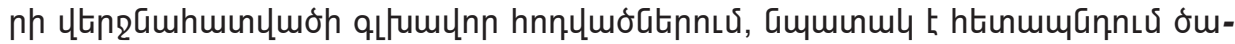

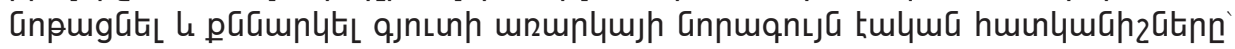

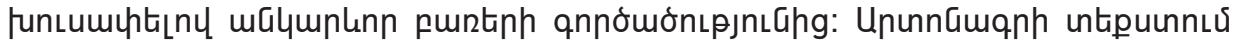

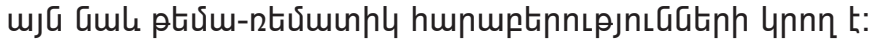

